\title{
Brisante Enthüllungen aus der Pharmaindustrie
}

\author{
Jürg Barben \\ PD Dr. med., Facharzt für Kinder und Jugendliche, speziell Pneumologie, FMH
}

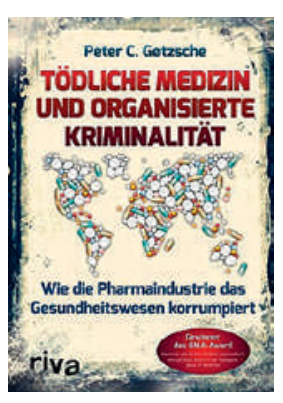

Peter C.Gøtzsche Tödliche Medizin und organisierte Kriminalität Wie die Pharmaindustrie das Gesundheitswesen korrumpiert

München: RIVAVerlag 2014

512 Seiten, $34.90 \mathrm{CHF}$. ISBN 978-3-86883-438-3
Es gibt Bücher, die werden von den Medien hochgelobt, damit sie möglichst viel gekauft und gelesen werden. Und dann gibt es Bücher, die einige lieber versteckt haben möchten, denn sie enthalten «intellektuellen Sprengstoff» und könnten ungeliebte heikle Diskussionen entfachen. Zur letzteren Kategorie gehört sicher Peter G $\varnothing$ tzsches Buch, das bisher nur wenigen Ärzten bekannt ist. Der provokative Buchtitel schreckt auf, verspricht jedoch eine spannende Lektüre mit vielen neuen Einsichten. Der Autor legt im Buch den Finger unverblühmt auf die heiklen Punkte in unserem Gesundheitswesen und zeigt auf, was die Pharmaindustrie alles unternimmt, um ihre Gewinne zu sichern. Neben der Manipulation von Studien und Unterschlagung von Studienergebnissen beschreibt der Autor auch die Aktivitäten und Marketing-Tricks der Pharmakonzerne, die weit über das Legale hinausgehen und die Ärzteschaft sowie zunehmend auch Patientenorganisationen im Visier haben.

Dabei vergleicht Gøtzsche die Machenschaften der Pharmaindustrie mit der Tabakindustrie, was aufhorchen lässt, und belegt diese Behauptung mit zahlreichen Dokumenten. Natürlich sehr zum Missfallen der Vereinigung Pharmafirmen in der Schweiz (VIPS), die

\section{Der Autor legt im Buch den Finger unverblühmt auf die heiklen Punkte im Gesundheitswesen.}

das Buch bereits als «unqualifizierte, polemische Rundumschlag» und "übles Machwerk» abgetan hat und eine inhaltliche Diskussion genauso scheut wie der Teufel das Weihwasser.

\section{Über den Autor}

Der Autor ist ein profunder Kenner der Materie. Gøtzsche ist Facharzt für Innere Medizin, hat viele Jahre für Pharmaunternehmen klinische Studien durchgeführt und sich dort um die Zulassung von Medikamenten gekümmert, war später Mitbegründer der Cochrane Collaboration und ist heute renommierter Professor für Forschungsdesign und Forschungsanalyse an der Universität Kopenhagen sowie Leiter des Nordic Cochrane Centre.

\section{Insider kritisieren}

Und Peter Gøtzsche ist nicht der erste, der die Pharmaindustrie mit der Tabakindustrie vergleicht. In seinem Buch zitiert er auch einen ehemaligen Vizepräsidenten von Pfizer mit den Worten: «Die Ähnlichkeit zwischen dieser Industrie und dem organisierten Verbrechen ist beängstigend: Die Mafia verdient unverschämt viel Geld, diese Industrie ebenfalls. Die Nebenwirkungen des organisieren Verbrechens sind Mord und Tote, und das sind auch die Nebenwirkungen dieser Industrie. Die Mafia besticht Politiker und andere Leute, die Pharmakonzerne tun das ebenfalls.» Unbestritten ist, dass die Pharmaindustrie sehr häufig mit dem Gesetz in Konflikt gerät und wiederholt zu Strafzahlungen in Milliardenhöhe verurteilt wird. Und trotzdem begeht sie immer wieder die gleichen Straftaten. Dabei geht es ganz profan um sehr viel Geld - vor allem auf Kosten der Gesundheit der Menschen. Im Vorwort schreibt Richard Smith, ehemaliger Herausgeber des British Medical Journal (BMJ) prägnant: «Die Industrie tötet viele Menschen, viel mehr als das organisierte Verbrechen. Hunderttausende sterben jedes Jahr an verschreibungspflichtigen Medikamenten. Vielen halten das für unvermeidlich, weil diese Arztneimittel gegen Krankheiten eingesetzt werden, die ihrerseits tödlich sind. Aber ein Gegenargument lautet: Der Nutzen der Medikamente wird übertrieben, und die Belege für ihre Wirksamkeit werden häufig gefälscht. Dieses ‘Verbrechen ' kann man der Industrie getrost vorwerfen.» So werden zum Beispiel Nebenwirkungen gezielt verschwiegen, wie beim Vioxx-Skandal von MSD vor 10 Jahren, als der COX-2-Hemmer Rofecoxib (Vioxx) Tausenden von Arthritis-Patienten den Tod gebracht hat. Peter Gøtzsche beschreibt schonungslos die Mechanismen und Manipulationen der Pharmaindustrie in der Zusammenarbeit mit Fachzeitschriften, Arzneimittelbehörden, Ärztschaft und Patientenorganisationen. Sein Buch gesellt sich zu Artikeln und Publikationen namhafter Ärzte, die in den letzten Jahren öffentlich Kritik an "Big Pharma» geäussert haben: "The Truth about the drug companies - how they deceive us and what to do about it" von Marcia Angell und "On the take - how medicine's complicity with big business can 
endanger your health" von Jerome Kassirer, beides ehemalige Herausgeber der renommierten Fachzeitschrift The New England Journal of Medicine sowie «The trouble with medical journals» von Richard Smith, ehemaliger Herausgeber des BMJ.

Besondere Kritik übt der Autor an der Tatsache, dass Pharmafirmen selber entscheiden dürfen, wie sie ein Medikament testen wollen, wie sie Daten auswerten und was sie davon an die offizielle Zulassungsbehörden oder der Öffentlichkeit weitergeben. Die Rohdaten werden dabei als unverrückbares Geschäftsgeheimnis gehütet. Die Fachzeitschriften ihrerseits publizieren teils unvollständige oder gar fehlerhafte Studien, damit Ihnen die Pharmaindustrie für viel Geld Sonderdrucke abkauft. So meinte Richard Horten, aktueller Herausgeber vom The Lancet, dass «Fachzeitschriften sich zu Informationswaschanlagen für die Pharmaindustrie entwickelt haben.» Die Sonderdrucke werden dann an die Ärzte verteilt, um sie über die Wirkung und Risiken von Medikamenten zu täuschen.

\section{Werbung vs. Korruption}

Im Bereiche der verschreibungspflichtigen Medikamente ist die Pharmaindustrie nach wie vor auf die $\mathrm{Zu}$ sammenarbeit mit den Ärzten angewiesen. Dazu wurden über Jahrzehnte ein feines Netz und Strukturen aufgebaut, die Ärzte dazu verleiten sollen, diese Medikamente auch zu verschreiben. Oder haben Sie als Arzt oder Ärztin noch nie ein Werbegeschenk der Pharmaindustrie angeboten bekommen? Eine Einladung für ein Fachreferat mit anschliessendem Abendessen? Oder eine gesponserte Reise zu einem Fachkongress? Oder einfach nur Medikamentenmuster zum ausprobieren oder Rabatte bei Bezug von grösseren Mengen? Oder eine Einladung zu einer post-marketing-Studie, deren Aufwand selbstverständlich vergütet wird? Natürlich stellt sich da die Frage: Wo hört «einfache` Werbung auf und wo beginnt die Korruption? Beim Verschenken von Kugelschreibern und post-it-Zettel, die den Alltag des Arztes erleichtern sollen? Oder erst beim «Kickback», der teilweisen Rückerstattung der bezahlten Gelder bei Medikamenten durch die Pharmaindustrie? Der Autor beschreibt diese Problematik derart detailliert, sodass sich anschliessend jeder Leser fragen muss: Wem ist der Arzt verpflichtet? Dem Patienten oder der Industrie? Geht es wirklich um das Wohl des Menschen oder um mehr Umsatz?

Peter Gøtzsche zeigt auch sehr zutreffend die zunehmenden Manipulationen der Patientenorganisationen durch die Pharmaindustrie auf: Offenbar sind die dort investierten Gelder heute viel effizienter angelegt als bei den Ärzten. Damit wird Druck von Patienten und deren Organisationen auf die Ärzte ausgeübt, wie wir es derzeit laufend bei der Einführung von Orphan Drugs bei seltenen Erkrankungen erleben.

\section{Lösungsvorschläge}

Das Buch handelt nicht nur von Missständen und Problemen, sondern Gøtzsche schlägt auch Lösungen vor, die eine breite öffentliche Diskussion verdienen. Ne-

\section{Geht es wirklich um das Wohl des Menschen oder um mehr Umsatz?}

ben einem (längst fälligen) Verbot für Direktwerbung von Medikamenten beim Konsumenten (in Presse, TV, Internet usw.) fordert Gøtzsche auch eine konsequente Offenlegung aller finanziellen Verbindungen zwischen der Pharmaindustrie und Ärzten, Fach- und Berufsverbänden, Patientengruppen sowie Fachzeitschriften. Ausserdem sollen alle Prüfpläne und Studiendaten, die für die Zulassung von Medikamenten notwendig sind, der Öffentlichkeit zugänglich gemacht und nicht mehr als Geschäftsgeheimnis der Industrie gehütet werden dürfen. Zudem fordert der Autor einen Systemwechsel bei der Medikamentenprüfung: Medikamentenstudien sollen durch unabhängige Forschergruppen und staatliche Institutionen durchgeführt werden. Das erfordert eine Stärkung der Unabhängigkeit der Institutionen, die Medikamente zulassen und überwachen. Da die grossen Gewinne in der Pharmaindustrie durch die Gelder der Allgemeinheit (u.a. mit Steuern und Krankenkassenbeiträgen) bezahlt werden, hat diese auch ein Anrecht zu erfahren, ob die Medikamente wirklich nützen oder die Gesundheit gefährden.

\section{Fazit}

Zusammenfassend handelt es sich um ein sehr lesenswertes und aufrüttelndes Buch über die Missstände im Gesundheitswesen. Es richtet sich nicht gegen den Fortschritt bzw. Erforschung und Entwicklung von unerlässlichen Medikamenten, sondern zeigt Fehlanreize und zunehmende Korruption im Gesundheitswesen auf. Ein Buch, das zum Nachdenken anregt und beschreibt, wie der zunehmend von Geldgier geprägte Gesundheitsmarkt die bisher bewährte ärztliche Heilkunst bedroht. Ein unerlässliches Buch für jede Ärztin und jeden Arzt, das im letzten Jahr auch Gewinner des British Medical Association's Annual Book Award wurde. 